\title{
Meynert nucleus-related cortical thinning in Parkinson's disease with mild cognitive impairment
}

\author{
Siming Rong", Yan Li", Bing Li", Kun Nie\#, Piao Zhang, Tongtong Cai, Mingjin Mei, Lijuan Wang, \\ Yuhu Zhang \\ Department of Neurology, Guangdong Neuroscience Institute, Guangdong Provincial People’s Hospital, Guangdong Academy of Medical Sciences, \\ Guangzhou, China \\ \#These authors contributed equally to this work.
}

Correspondence to: Yuhu Zhang, MD; Lijuan Wang, MD, PhD. Department of Neurology, Guangdong Neuroscience Institute, Guangdong Provincial People's Hospital, Guangdong Academy of Medical Sciences, No. 106 Zhongshan Er Road, Guangzhou 510080, China.

Email: yhzhangsd@126.com; wljgd68@163.com.

Background: Cognitive impairment in Parkinson's disease (PD) involves the cholinergic system and cholinergic neurons, especially the nucleus basalis of Meynert (NBM/Ch4) located in the basal forebrain (BF). We analyzed associations between NBM/Ch4 volume and cortical thickness to determine whether the NBM/Ch4-innervated neocortex shows parallel atrophy with the NBM/Ch4 as disease progresses in PD patients with cognitive impairment (PD-MCI).

Methods: We enrolled 35 PD-MCI patients, 48 PD patients with normal cognition (PD-NC), and 33 age- and education-matched healthy controls (HCs), with all participants undergoing neuropsychological assessment and structural magnetic resonance imaging (MRI). Correlation analyses between NBM/Ch4 volume and cortical thickness and correlation coefficient comparisons were conducted within and across groups.

Results: In the PD-MCI group, NBM/Ch4 volume was positively correlated with cortical thickness in the bilateral posterior cingulate, parietal, and frontal and left insular regions. Based on correlation coefficient comparisons, the atrophy of NBM/Ch4 was more correlated with the cortical thickness of right posterior cingulate and precuneus, anterior cingulate and medial orbitofrontal lobe in PD-MCI versus HC, and the right medial orbitofrontal lobe and anterior cingulate in PD-NC versus HC. Further partial correlations between cortical thickness and NBM/Ch4 volume were significant in the right medial orbitofrontal (PDNC: $r=0.3, P=0.045$; PD-MCI: $r=0.51, \mathrm{P}=0.003$ ) and anterior cingulate (PD-NC: $r=0.41, \mathrm{P}=0.006$; PD-MCI: $\mathrm{r}=0.43, \mathrm{P}=0.013)$ in the $\mathrm{PD}$ groups and in the right precuneus $(\mathrm{r}=0.37, \mathrm{P}=0.04)$ and posterior cingulate $(\mathrm{r}=0.46$, $\mathrm{P}=0.008$ ) in the PD-MCI group.

Conclusions: The stronger correlation between NBM/Ch4 and cortical thinning in PD-MCI patients suggests that NBM/Ch4 volume loss may play an important role in PD cognitive impairment.

Keywords: Parkinson's disease (PD); cholinergic system; cognitive impairment; nucleus basalis of Meynert; cortical thickness

Submitted Mar 15, 2020. Accepted for publication Nov 24, 2020.

doi: 10.21037/qims-20-444

View this article at: http://dx.doi.org/10.21037/qims-20-444 


\section{Introduction}

Parkinson's disease (PD) is a neurodegenerative disease characterized by typical motor symptoms such as bradykinesia, resting tremor, and rigidity and is accompanied by nonmotor symptoms including cognitive impairment, anxiety, depression, and sleep disorders (1). Up to $80 \%$ of patients with PD will develop dementia in the late stage, which may greatly increase the cost of medical resources and reduce quality of life $(2,3)$. Cognitive impairment in $\mathrm{PD}$ is the consequence of multifactorial interactions, such as dysfunction in neurotransmission and the deposition of $\alpha$-synuclein and Alzheimer-type pathology (4). Neurotransmitter systems, especially cholinergic and dopaminergic systems, are disrupted by pathological damage, such as $\alpha$-synuclein and Alzheimer-type pathology, and contribute to cognitive deterioration (5). Both neurotransmitters play roles in $\mathrm{PD}$ cognitive impairment; moreover, the dual syndrome hypothesis provides an explanatory model for neurotransmitter-related cognitive impairment, which involves dopaminergic deficits in frontostriatal executive dysfunction and other transmitters dysfunction, especially cholinergic dysfunction (6). Based on the dual syndrome hypothesis, cholinergic dysfunction would probably lead to dementia.

In the brain, the cholinergic system includes two anatomically distinct aggregations of cholinergic neurons $(\mathrm{Ch})$; the pedunculopontine complex (PPN), which innervates the thalamus, striatum, cerebellum, and brainstem for the regulation of motor function, and the basal forebrain (BF), which projects fibers to the neocortex, posterior cingulate, amygdala, and hippocampus for the regulation of cognitive function (7). In the $\mathrm{BF}$, Ch can be anatomically divided into four parts: $\mathrm{Ch} 1$ and $\mathrm{Ch} 2$ project to the hippocampus, $\mathrm{Ch} 3$ projects to the olfactory bulb, and the nucleus basalis of Meynert (NBM/Ch4) projects to the neocortex and the amygdala (8). With the progression of cognitive decline, $\mathrm{BF}$ projecting regions including the hippocampus $(9,10)$ and neocortex $(11,12)$ were found to be degenerative in $\mathrm{PD}$ with dementia (PDD). However, while NBM/Ch4 Ch showed more severe $\alpha$-synuclein pathology and cell loss in PDD, Ch1 and Ch2 cholinergic septal neurons were spared from pathological deposits of $\alpha$-synuclein (13). NBM/Ch4 was also shown to be damaged by the deposit of $\alpha$-synuclein at Braak stage IV and was accompanied by motor symptoms (14) indicating NBM/ $\mathrm{Ch} 4$ degeneration is one of the specific pathological changes in $\mathrm{PD}$ with cognitive impairment.
Studies show that NBM/Ch4 is the major source of cholinergic projections to the neocortex and amygdala (15), and its pathological atrophy has been confirmed by in vivo magnetic resonance imaging (MRI) evidence of reduced $\mathrm{BF}$ volume in patients with mild cognitive impairment (MCI) $(16,17)$. Although volume loss in the substantia innominata can be present in PD patients with intact cognition, greater loss is seen in those who progress from PD-MCI to PDD $(18,19)$. Furthermore, NBM/Ch4 volume loss was demonstrated to be a predictor of future cognitive decline (20-22). While these studies may have uncovered the status of NBM/Ch4 in PD cognitive impairment, whether changes occur in NBM/Ch4-innervated regions in PD remains unknown. Diffusion and functional studies have found that structural and functional connections are decreased between the $\mathrm{Ch} 4$ and the neocortex in PD (23) suggesting the cortical region involving innervation from the NBM/Ch4 may have changed along with damage to the projection fibers.

Cortical thinning is a marker of MCI (24) and PD-MCI (25-27) and is severe in PDD (28-30). Positron emission tomography (PET) tracers of acetylcholinesterase (AChE) activity in PD patients show cortical cholinergic deficits become more widespread and profound as cognition deteriorates and always occur in the posterior region, including the temporoparietal and occipital regions $(31,32)$. As the posterior cortex receives projections of cholinergic fibers from the NBM/Ch4 (33), a cholinergic deficit may lead to posterior dysfunction and finally result in cortical thinning. A previous vertex-wise study found stronger correlations between temporal lobe cortical thickness and variations in NBM/Ch4 volume in amnestic MCI (aMCI) patients than in healthy controls (HCs) (34). Furthermore, the regions innervated by the NBM/Ch4 in humans are more extensive than those observed in animal studies and involve the temporal, parahippocampal, cingulate, and temporal polar regions (35). However, these correlations are not well defined in PD patients with cognitive impairment. We hypothesize that NBM/Ch4 volume loss is correlated with cortical thinning of its innervated regions, especially the posterior cortex in PD patients, and this study thus aimed to analyze the relationship between NBM/Ch4 volume loss and cortical thickness in PD-MCI patients.

\section{Methods}

\section{Participants}

Participants in this prospective study were recruited from 
the Department of Neurology, Guangdong Neuroscience Institute, Guangdong Provincial People's Hospital, Guangzhou, China, from October 2015 to December 2019, and included 83 PD patients and 33 age- and educationmatched HCs. PD was clinically diagnosed following the Queen Square Brain Bank criteria, and PD subjects met the following criteria: age between 40 and 80 years, without the presence of dementia according to the Movement Disorders Society criteria (36), Hoehn and Yahr stage $\leq 3$, and no severe depression or anxiety [Hamilton Depression Scale (HAMD) $\leq 35$ points and Hamilton Anxiety Scale (HAMA) $\leq 29$ points). The medical treatment of patients included levodopa, dopaminergic agonists, monoamine oxidase inhibitors, and catechol-O-methyltransferase inhibitors. Those with a medication history of acetylcholinesterase inhibitors were excluded from the study. Volunteers with no neurological or mental illness and without a recent medication history of acetylcholinesterase inhibitor use were rated by MiniMental State Examination (MMSE) and Montreal Cognitive Assessment (MoCA). Those with a MoCA score higher than the educational corrected cutoff value of the general Chinese population was chosen as HCs (37). All subjects provided written informed consent to the research protocol, which was approved by the Medical Ethics Committee of Guangdong Provincial People's Hospital [no. GDREC2018338H(R1)].

\section{Clinical and neuropsychological evaluation}

The motor function of most subjects was evaluated by the Movement Disorder Society-sponsored Revision of the Unified Parkinson's Disease Rating Scale part III (MDS-UPDRS-III) under the "ON" condition, while the remaining subjects were assessed by the Unified Parkinson's Disease Rating Scale part III (UPDRS-III). The UPDRSIII score was transformed by the formula used to transform UPDRS-III scores into MDS-UPDRS-III scores (38). The neurobehavioral assessment included the HAMA and HAMD. The neuropsychological assessments of our study were as follows: MMSE and MoCA for the assessment of global cognitive efficiency; the animal fluency test for executive functions, digit span and digit symbol tests for attention and working memory; logical memory and immediate memory tests from the Wechsler Memory Scale (WMS) and vocabulary and similarities subtests (WAIS-R) for language; and block design and object assembly for visuospatial function. Patients with PD were divided into $\mathrm{PD}$ with normal cognition (PD-NC) and PD with mild cognitive impairment (PD-MCI) groups according to the Level I criteria of the Movement Disorder Society (MDS) Task Force guidelines (39). The identification of PD-MCI was based on the criteria of a score $\geq 1.5$ standard deviation (SD) below the mean in at least two tests in five domains $(40,41)$.

\section{Neuroimaging}

\section{MRI data acquisition}

To acquire MRI data a GE 3.0 T MR imaging system (GE Healthcare, Fairfield, CT, USA) was used. All subjects underwent three-dimensional spoiled gradient recalled (3D-SPGR) sequences according to the following parameters: number of scanning slices, 146; slice thickness, $1.00 \mathrm{~mm}$; slice spacing, $1.00 \mathrm{~mm}$; matrix, 256×256; field of view (FOV), $512 \times 512$; voxel, $0.469 \times 0.469 \times 1.0 \mathrm{~mm}^{3}$; flip angle, $13^{\circ}$; repetition time (TR), $7.6 \mathrm{~ms}$; and echo time (TE), $3.3 \mathrm{~ms}$.

\section{Volume estimation of the BF}

The structural MRI of each participant was checked visually to ensure the absence of motion artefact. Structural MRI data were preprocessed using the VBM8 (Voxel-based morphometry) (http://www.neuro.unijena.de/vbm/download/) approach integrated in SPM8 (Wellcome Trust Centre for Neuroimaging; www.fil.ion. ucl.ac.uk/spm) and implemented in MATLAB R2018b (MathWorks, Natick, MA, USA). Structural MRI data were automatically segmented into gray matter, white matter (WM), and cerebrospinal fluid (CSF) partitions of $1.5-\mathrm{mm}$ isotropic voxel size using the segmentation routine of the VBM8 toolbox. The segmented gray matter and WM were obtained and high-dimensionally registered to Montreal Neurological Institute (MNI) space by using the diffeomorphic anatomical registration through an exponentiated lie algebra (DARTEL) algorithm (42). The segmented image of each subject was rechecked visually to ensure the accuracy of registration. The modulated nonlinear-warping GM image was then used to extract the volume of the NBM/Ch4.

The mask of the region of interest (ROI) for the NBM/ Ch4 was identified using cytoarchitectonic mapping implemented in the SPM Anatomy Toolbox $(43,44)$ to ensure anatomical precision and replicability. The BF is composed of a magnocellular cholinergic cell complex and has been defined histologically in primates as Ch1Ch4 (15). A probabilistic anatomical map of the BF was 
obtained from a postmortem human brain, and a stereotaxic map has recently been made available (44). The NBM/ $\mathrm{Ch} 4$ is one of the regions in the $\mathrm{BF}$, and the NBM/Ch4 volume of each subject was calculated by summing up the bilateral voxel values within the masks of the ROI from the modulated gray matter without smoothing (45). The NBM/ Ch4 volume was then normalized across all subjects by the following algorithm: normalized volume $=$ total volume $\left(\mathrm{mm}^{3}\right) /$ total intracranial volume $(\mathrm{TIV})\left(\mathrm{mm}^{3}\right) \times 1,000$. The TIV was calculated as the sum of the total segmented gray matter, WM, and CSF volumes.

\section{Cortical thickness estimation}

Structural MRI data were preprocessed using the analysis pipeline of FreeSurfer v.5.3 (http://surfer.nmr.mgh. harvard.edu/). Image preprocessing included the following steps: magnetic field inhomogeneity correction, motion correction, removal of nonbrain tissues such as the scalp and skull, automated Talairach transformation, segmentation of the GM and WM boundaries, automatic topological correction and registration with standard brain templates, Gaussian smoothing, reconstruction of the bilateral cerebral cortex, and calculation of the cortical thickness. Cortical thickness was calculated as the closest distance from the gray matter/WM boundary to the gray matter/CSF boundary at each vertex. A general linear model (GLM) was assessed for statistical analysis in the Query, Design, Estimate, Contrast (Qdec) module of FreeSurfer with NBM/Ch4 volume on the horizontal axis and cortical thickness on the vertical axis. Vertex-wise analyses were performed in each group to investigate whether the volume variation in the NBM/Ch4 was correlated with a variation in cortical thickness. We also compared the slope from the GLM in cortical thickness across each group to investigate the group differences in correlation coefficients. Monte Carlo simulation was applied to provide cluster-wise correction for multiple comparisons, and the cluster-wise probability significance level of the results was set to 0.05 .

\section{Statistical analysis}

Statistical analysis was performed using SPSS Statistics version 25.0 (IBM SPSS Statistics, Armonk, NY, USA). The Kolmogorov-Smirnov test was used to assess a normal distribution, and the normally distributed continuous variables were then compared by analysis of covariance (ANCOVA) and unpaired $t$-tests, while the Kruskal-Wallis $\mathrm{H}$ test and Mann-Whitney $U$ test were used for nonnormally distributed parameters. The chi-square test was used to compare categorical variables. Pearson's correlation was performed in the HC and PD groups to determine the correlation coefficients between NBM/Ch4 volume and other parameters. We found that the normalized volume of the NBM/Ch4 correlated with the duration of disease $(r=-0.436, P=0.009)$ in the PD-MCI group and age $(r=-0.466$, $\mathrm{P}=0.001)$ in the PD-NC group. Sex differences in structural changes were also assessed in each group, and the normalized volume of the NBM/Ch4 was smaller in males. Thus, we applied age, sex, and duration of disease as nuisance factors in correlation analyses. We additionally investigated the effect of NBM/Ch4 in each group by using partial correlation analysis between the normalized volume of the NBM/Ch4 and the average thickness of the significant region (right medial orbitofrontal, anterior cingulate, posterior cingulate and precuneus) in the comparison of correlation efficiencies across groups. The age, duration of disease, and sex were also included in the correction as cofactors.

\section{Results}

\section{Demographic and clinical characteristics}

Demographic and clinical characteristics, along with normalized volumes of the NBM/Ch4 and TIV, are shown in Table 1. The results of the neuropsychological battery are shown in Table S1. No significant differences were noted among the PD-NC, PD-MCI and HC groups in age, sex, education, HAMA, HAMD, or TIV. A significant difference was found in MMSE ( $\mathrm{H}=8.59, \mathrm{P}=0.014), \mathrm{MoCA}(\mathrm{F}=17.75$, $\mathrm{P}<0.001)$, and normalized volume of $\mathrm{Ch} 4 / \mathrm{NBM}(\mathrm{F}=3.33$, $\mathrm{P}=0.39$ ) across the three groups. Bonferroni post hoc test showed that HC had greater performance on MMSE $(\mathrm{P}=0.018)$; Tukey pair-wise tests revealed PD-MCI had a worse performance on MoCA than $\mathrm{PD}-\mathrm{NC}(\mathrm{P}=0.002)$ and $\mathrm{HC}(\mathrm{P}<0.001)$ and better performance in $\mathrm{HC}$ than $\mathrm{PD}-\mathrm{NC}$ $(\mathrm{P}=0.012)$. Both PD-NC $(\mathrm{P}=0.057)$ and PD-MCI $(\mathrm{P}=0.072)$ tended to have a smaller normalized volume of NBM/Ch4 than HC. In the comparison of the PD-NC and PD-MCI groups, duration of disease, H-Y stage, MDS-UPDRS-III, and levodopa equivalent daily dose showed no significant difference (Table 1).

\section{Structural correlations in PD-MCI, PD-NC, and HC subjects}

Brain regions with significant correlations between NBM/ 
Table 1 Demographics and clinical characteristics

\begin{tabular}{|c|c|c|c|c|c|}
\hline Variable & PD-NC & PD-MCl & $\mathrm{HC}$ & Test stat & $P$ value $(P<0.05)$ \\
\hline Age (yrs) & $58.61(7.45)$ & $60.40(8.51)$ & $58.36(7.25)$ & $0.897^{\mathrm{a}}$ & 0.411 \\
\hline Sex (female/male) & $16 / 32$ & $18 / 17$ & $18 / 15$ & $4.44^{\mathrm{b}}$ & 0.11 \\
\hline Education (yrs) & $10.21(3.53)$ & $8.9(3.9)$ & $9.88(3.37)$ & $2.27^{c}$ & 0.32 \\
\hline MoCA & $23.98(3.52)$ & $21.23(4.33)$ & $26.27(2.25)$ & $17.75^{\mathrm{a}}$ & $<0.001^{*}$ \\
\hline HAMA & $7.68(6.93)$ & $7.26(6.0)$ & $6.09(5.81)$ & $1.30^{\mathrm{c}}$ & 0.52 \\
\hline HAMD & $8.02(6.72)$ & $8.0(6.96)$ & $6.27(7.93)$ & $4.19^{c}$ & 0.12 \\
\hline Duration of disease (yrs) & $3.05(2.29)$ & $3.27(2.58)$ & - & $-0.061^{d}$ & 0.95 \\
\hline LEDD (mg) & $194.2(196.6)$ & $179.5(221.5)$ & - & $-0.54^{d}$ & 0.59 \\
\hline Normalized volume of NBM/Ch4 & $0.0995(0.023)$ & $0.099(0.029)$ & $0.1155(0.041)$ & $3.33^{a}$ & $0.039^{*}$ \\
\hline $\operatorname{TIV}\left(\mathrm{mm}^{3}\right)$ & $1,333.33(120.57)$ & 1,345.69 (117.49) & $1,302.81(128.19)$ & $1.12^{\mathrm{a}}$ & 0.33 \\
\hline
\end{tabular}

${ }^{a}$, one-way analysis of variance; ${ }^{b}$, Pearson's chi-square $\left(\chi^{2}\right)$; ${ }^{c}$, Kruskal-Wallis $\mathrm{H}$ test; ${ }^{\mathrm{d}}$, Mann-Whitney $\mathrm{U}$ test; ${ }^{\mathrm{e}}$, Student's $t$-test. Data are presented as mean and standard deviation. MMSE, Mini-Mental State Examination; MoCA, Montreal Cognitive Assessment; HAMA, Hamilton Anxiety Scale; HAMD, Hamilton Depression Scale; HY, Hoehn and Yahr scale; MDS-UPDRS-III, part III of the Movement Disorder Society-sponsored Revision of the Unified Parkinson's Disease Rating Scale; LEDD, levodopa equivalent daily dose.

Ch4 and cortical thickness were found in the PD-MCI group (Figure 1 and Table S2). After correcting for age, sex, and disease duration, the left posterior cingulate gyrus (Brodmann 31), pars opercularis gyrus (insula, Brodmann 44), precuneus (parietal lobe, Brodmann 7, 19), superior frontal lobe (frontal lobe, Brodmann 10, 32), right posterior cingulate (Brodmann 23, 31), superior parietal (parietal lobe, Brodmann 7), and superior frontal lobe (frontal lobe, Brodmann 10, 11, 12) had significant correlations between the volumetric variations in the NBM/Ch4 and cortical thickness in the PD-MCI group. However, there was no significant correlations between regions in the PD-NC and HC groups. Volumetric variations in the $\mathrm{NBM} / \mathrm{Ch} 4$ were not correlated with the MMSE, MoCA, or the tests of each domain in the PD-MCI, PD-NC, and HC groups. Otherwise, no significant differences were revealed when the cortical thickness across groups was compared.

\section{Group differences in correlation coefficients across the PD- NC, PD-MCI, and HC groups}

Stronger correlations between volumetric variations in the
NBM/Ch4 and cortical thickness were found in the right medial orbitofrontal lobe, anterior cingulate gyri, posterior cingulate gyri, and precuneus in comparisons between the PD-MCI and HC groups. However, in comparisons between the PD-NC and HC groups, the right medial orbitofrontal lobe and right anterior cingulate gyri showed stronger correlations (Figure 2 and Table S3). No significant differences were found in comparisons of the $\mathrm{PD}-\mathrm{NC}$ and PD-MCI groups. The effect of NBM/Ch4 volume on the extracted average cortical thickness of the regions mentioned above was conducted by using partial correlation analysis and showed significant correlations between $\mathrm{PD}-\mathrm{NC}$ in the right medial orbitofrontal $(\mathrm{r}=0.3, \mathrm{P}=0.045)$ and anterior cingulate $(\mathrm{r}=0.41$, $\mathrm{P}=0.006)$. However, in PD-MCI groups, the right medial orbitofrontal $(\mathrm{r}=0.51, \mathrm{P}=0.003)$, anterior cingulate $(\mathrm{r}=0.43$, $\mathrm{P}=0.013)$, right precuneus $(\mathrm{r}=0.37, \mathrm{P}=0.04)$, and posterior cingulate ( $\mathrm{r}=0.46, \mathrm{P}=0.008$ ) were found to be significantly correlated with NBM/Ch4 volume only in the PD-MCI group, and no significant associations in these regions were noted in the HC group. 

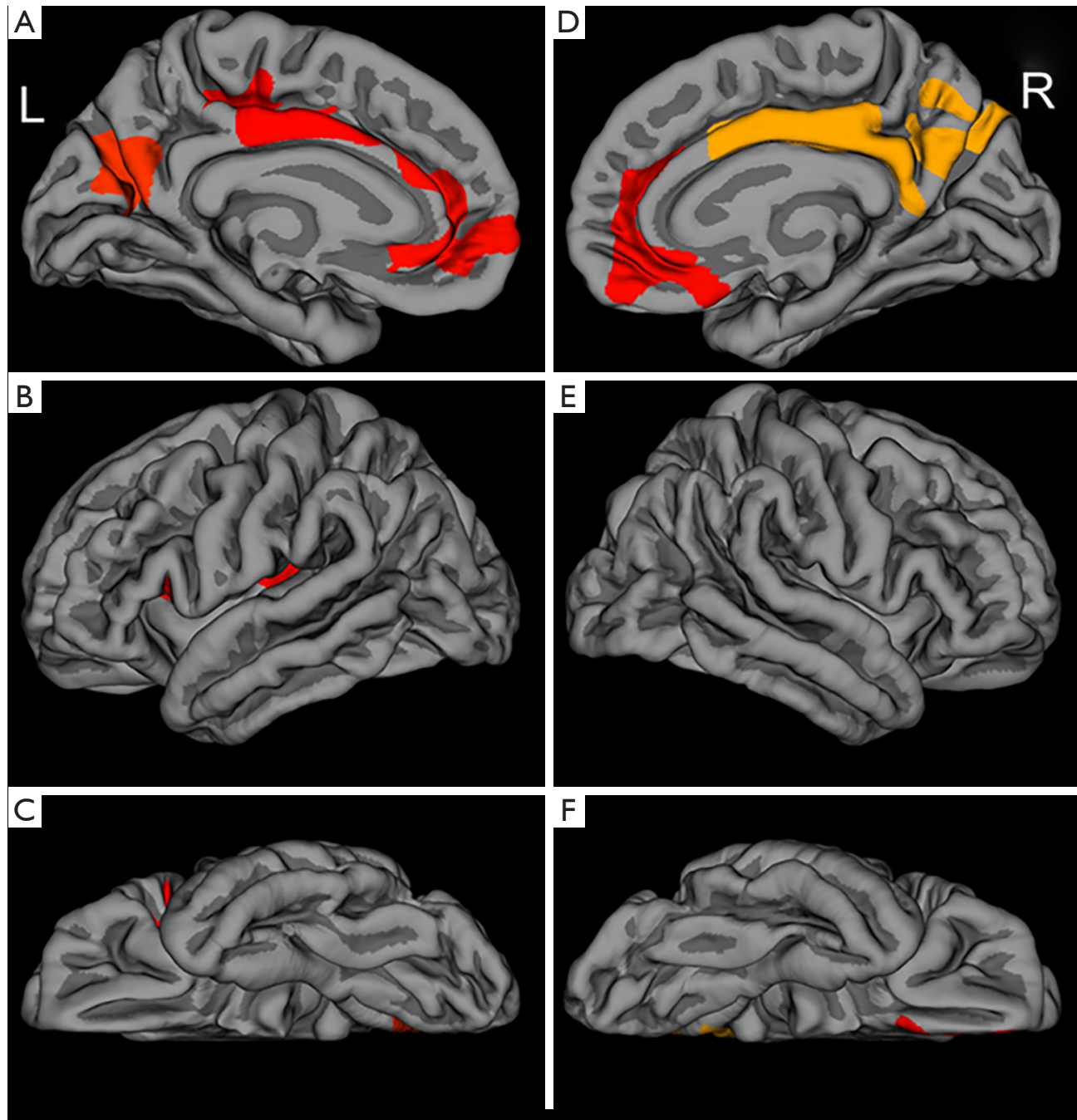

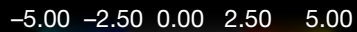

Figure 1 The regions with significant positive correlation between the volume of the NBM/Ch4 and cortical thickness in PD-MCI patients (correcting for sex, age and duration of disease). (A,B) Lateral aspect; (B,E) medial aspect; (C,F) inferior aspect. The red-orange areas highlight areas of significant positive correlation. The color bar shows the logarithmic scale of $\mathrm{P}$ values $(-\log 10)$.

\section{Discussion}

The mechanisms underlying cognitive impairment in PD are unclear but have always been considered a consequence of multifactorial interactions. Cholinergic deficits were considered as one of the pivotal factors in these interactions, and atrophy in the NBM/Ch4 has been regarded as one of the markers of these deficits. The present study was first to investigate the relationship between NBM/Ch4 volume and cortical thickness in PD patients with different cognitive states. The major findings were as follows: (I) volume differences in the NBM/Ch4 were correlated with the thickness of the bilateral posterior cingulate gyrus, parietal lobe, frontal lobe and left insula lobe in PD-MCI patients; (II) PD-MCI patients showed a stronger correlation between NBM/Ch4 volume loss and cortical thickness in the right medial orbitofrontal, anterior cingulate, precuneus, and posterior cingulate than the HCs, and only the right medial orbitofrontal and anterior cingulate showed a difference in contrast between the PD-NC and HC groups. These findings suggested that NBM/Ch4 volume loss had a varying association with different cognitive states in $\mathrm{PD}$ patients and that the NBM/Ch4 affected the cingulate, 

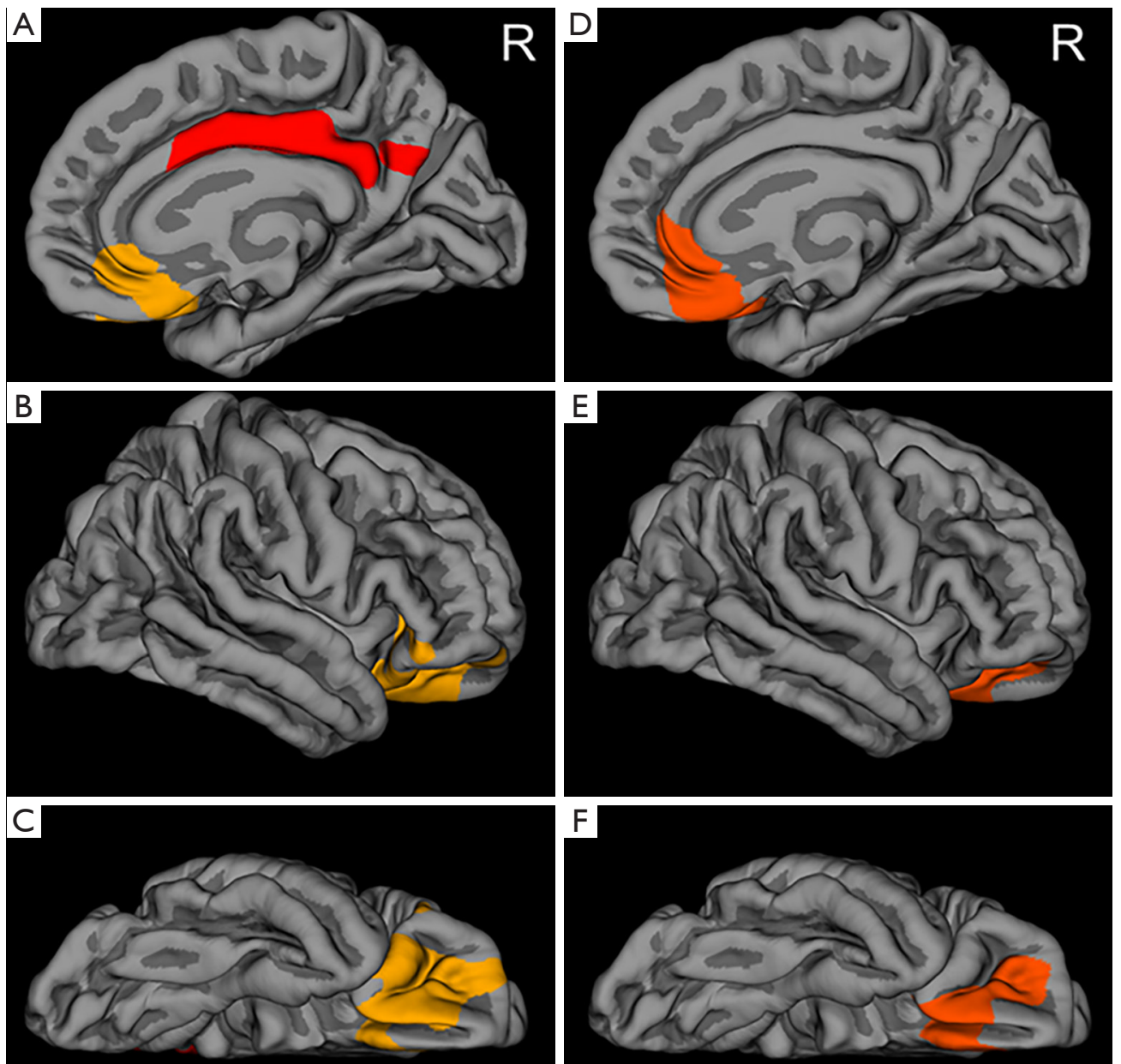

$\mathrm{PD}-\mathrm{MCl}$ vs $\mathrm{HC}$

PD-NC vs HC

$\begin{array}{lllll}-5.00 & -2.50 & 0.00 & 2.50 & 5.00\end{array}$

Figure 2 The regions with significantly stronger correlations between the volume of the NBM/Ch4 and cortical thickness in the comparison of the PD-MCI versus HC groups (left) and the PD-NC versus HC groups (right). (A,D) Medial aspect; (B,E) lateral aspect; (C,E) inferior aspect. The red-orange areas highlight areas of significance. The color bar shows the logarithmic scale of $\mathrm{P}$ values (-log10).

orbitofrontal, and precuneus regions.

Our study revealed a correlation between NBM/Ch4 volume loss and the cortical thickness of certain regions including the frontal lobe, insular lobe, parietal lobe, and cingulate lobe in PD-MCI patients but not in PD-NC patients and HCs. Pathology studies have suggested that as the main source of cholinergic projections, NBM $\backslash \mathrm{Ch} 4$ widely projects cholinergic fibers through the cingulate gyri to the amygdala and neocortex. Moreover, a previous study found subdivisions of the Ch4/NBM projecting fibers to certain cortical regions (15). However, due to the lack of clear anatomical borders between different NBM/Ch4 cell regions on MRI scans, the borders between subdivisions have not been accurately delineated, and we did not perform correlation analyses between them and cortical thickness on this basis. In a previous pathological study, Mufson et al. found cell loss in NBM/Ch4 restricted to the subdivisions of the anterior-lateral part (Ch4al) and posterior part $(\mathrm{Ch} 4 \mathrm{p})$ in $\mathrm{PD}$ and accompanied by a reduction of the Ach fiber plexus in the temporal lobe and amygdala (46). Hence, the cell loss of Ch4 may lead to a decrease of AchE fibers and cortical thinning. However, some radionuclide imaging 
studies found extensive reduction of AchE activity not only in the temporal lobe and amygdala but also in the occipital and global cerebrum $(32,47)$. Therefore, other subdivisions of Ch4 might also influence cortical AchE activity and play a role in cortical thinning in PD.

Previous structural imaging studies have found more extensive cortical thinning in PDD compared with PD$\mathrm{MCI}$ and that the progression from PD-MCI to PDD is gradual and subtle. Furthermore, not all PD-MCI patients progressed to PDD. This suggests cortical thinning may be initiated and progressively worsened by factors such as transmitter dysfunction and Alzheimer-type damage. Degeneration of the NBM/Ch4 ranged from $32 \%$ cell loss in nondemented PD patients to $54-70 \%$ in PDD patients $(13,48)$, and recent studies have found that a loss in $\mathrm{NBM} / \mathrm{Ch} 4$ volume could predict $\mathrm{PD}$-associated cognitive impairment (20-22). In our study, the thickness of cortical regions innervated by the NBM/Ch4 were correlated with volume changes in the NBM/Ch4 in PD-MCI patients. Based on the typical "bottom-up" pathological hypothesis in $\mathrm{PD}$, which describes the spread of $\alpha$-synuclein in PD starting from the olfactory bulb, substantia nigra, midbrain, and $\mathrm{BF}$ to the extensive neocortex (14), we speculate that PD-MCI patients with NBM/Ch4 volume loss tend to develop cortical thinning and finally progress to PDD. However, the cross-sectional nature of our study could not explain the causality; that is, whether cholinergic denervation from cholinergic $\mathrm{BF}$ degeneration leads to decreased cortical thickness remains unclear. Furthermore, only PD patients in the early stage and not those with advanced PD or PDD were enrolled in our study, and follow-up studies or enlargement of the sample is therefore needed to verify our perspective.

According to the results of the correlation coefficient comparisons among each group, no difference was found between the PD groups. When compared with HC, the region with significant difference in $\mathrm{PD}$-MCI was more extensive than that in PD-NC. A stronger correlation between the cortical thickness of the right orbitofrontal lobe (medial orbitofrontal lobe and anterior cingulate gyri), posterior cingulate gyri, and precuneus with NBM/ Ch4 atrophy was found in PD-MCI compared with HC. However, comparison between PD-NC and HC showed only the right orbitofrontal lobe (medial orbitofrontal lobe and anterior cingulate gyri) to have a stronger correlation with NBM/Ch4 atrophy in PD-NC. As NBM/Ch4 atrophy is one of the markers of cholinergic deficit, cortical thickness in the right medial orbitofrontal and anterior cingulate, posterior cingulate, and precuneus of $\mathrm{PD}-\mathrm{MCI}$ may be more susceptible to cholinergic deficit. Otherwise, the significant region in the comparison between PD-MCI and $\mathrm{HC}$ is more extensive than the result of the comparison between PD-NC and HC; therefore, this result suggested that PD-MCI could be more greatly influenced by NBM/ $\mathrm{Ch} 4$ volume loss than PD-NC. The partial correlation analyses showed regions of the right posterior cingulate and precuneus were significant only in the PD-MCI group. A previous diffusion tensor imaging study suggested that the cingulate lobe was part of the pathway of fibers from the $\mathrm{NBM} / \mathrm{Ch} 4$, which then projected to the posterior cortex including the temporoparietal and occipital regions (49). This suggests that the discrepancy between the PD-NC and PD-MCI groups in the correlations of NBM thickness could be attributed to the dysfunction of the posterior cingulate. According to our result, posterior cingulate and precuneus are part of the parietal-temporal area, which is located in the posterior cortex, and dysfunction in these regions has been shown to predict cognitive impairment in PD patients (50-52). Atrophy of the posterior cingulate gyri (29) and precuneus lobe has been regarded as a marker of PDD, along with reduced metabolism $(53,54)$ and AChE activity (47). Hypometabolism of the cortex has consistently been found to precede atrophy in PD patients with cognitive impairment (55). Our results show cortical thinning of the posterior cingulate and precuneus was more strongly correlated with NBM/Ch4 atrophy in PDMCI which in turn was correlated with cortical cholinergic deficit. On this basis, we speculated that NBM/Ch4 volume loss would lead to hypometabolism and cortical cholinergic deficits in the posterior cingulate and precuneus and initiate cortical thinning of these regions in the early stages of cognitive impairment.

In addition to the above, the thickness of the medial orbitofrontal lobe and the anterior cingulate was correlated with NBM/Ch4 volume loss in both the PD-MCI and PD$\mathrm{NC}$ groups but not the HC group. The medial orbitofrontal lobe, anterior cingulate gyri, and associative circuits in the prefrontal cortex correspond to the frontal-striatal loop, which is a part of the dopaminergic network (56), and cortical atrophy in the medial orbitofrontal lobe and anterior cingulate is widely recognized as a marker of dopaminergic deficit-regulated executive and attentional dysfunction in PD. A previous study revealed that efferent projections from the NBM/Ch4 also arrive at the orbitofrontal lobe (15) as part of the lateral orbitofrontal circuit, which is a part of the frontal-striatal loop, and then pass through the anterior 
cingulate (56). Radionuclide imaging studies have found the medial orbitofrontal lobe and anterior cingulate to have reduced dopaminergic activity $(32,57)$, but no cholinergic deficit or hypometabolism in PD-MCI and PDD patients was found when compared with healthy aging controls $(47,53,55)$. Therefore, we speculate that the NBM/Ch4 might regulate dopaminergic function, which is the typical dysfunction associated with PD, through its own efferent fibers. Previous Korean studies have suggested that a smaller volume of the substantia innominate and frontal atrophy at baseline can distinguish between those who progress from PD-MCI to PDD and those who do not $(18,58)$. In our study, the frontal lobe regions in both the PD-NC and PDMCI groups were affected by NBM/Ch4 volume, which implies that atrophy of the frontal lobe was accompanied by NBM/Ch4 volume loss, which eventually becomes a marker of PDD conversion. Therefore, we believe that the NBM/ Ch4 not only regulates the cholinergic system in PD but also plays an important role in the dopaminergic system.

It is widely recognized that cognitive impairment in PD begins in the subcortical regions because of its "bottomup" pathological pattern. Recent in vivo studies found that NBM/Ch4 volume loss, which reflects neuron loss, can predict cognitive impairment in PD patients $(18,20)$. However, in the early stage of $\mathrm{PD}, \mathrm{NBM} / \mathrm{Ch} 4$ volume loss was not correlated with cognitive performance at baseline $(18,20)$ and was not different in PD-MCI and PD-NC groups (19), which was consistent with the results of this study. However, some diffusion imaging and functional studies found that $\mathrm{PD}$ patients with cognitive impairment had increased mean diffusivity (MD) in the NBM/Ch4 (21) and decreased structural integrity and connectivity correlated with cognitive performance at baseline (23). Presumably, NBM/Ch4 volume loss is preceded by damage to its microstructure, and thus NBM/Ch4 volume loss may not influence cognition at baseline. Clinically, a previous DBS study targeting the NBM in AD showed less advanced cortical atrophy in the cortical thickness of the frontoparietal-temporal regions (59), and animal studies have suggested that stimulating the NBM can induce neural vasodilatation and increase cerebral blood flow in the cortex (60). Therefore, DBS targeting NBM/Ch4 may relieve cognitive impairment in $\mathrm{PD}$, but more studies and clinical trials are needed to verify the effects in PD-MCI .

Previous in vivo MRI studies investigating cortical thickness between PD-MCI, PD-NC, and HC groups have found that the pattern of cortical thinning in PDMCI patients included the frontal, insular, and parietal- temporal regions $(25-27,61)$. However, we found no significant differences in cortical thinning across groups; and similar results were also reported in some studies with small sample sizes $(62,63)$. Otherwise, the mean duration of PD was approximately 3 years, the average age of the groups was approximately 60 years, and individuals with these characteristics tended to have mild cortical changes $(29,64)$. Thus, these reasons may take account of why there was no significant difference in the comparison of cortical thickness.

\section{Conclusions}

The results of this study indicate that volume changes in the NBM/Ch4, which are involved in cholinergic deficits, might play different roles in different cognitive states, especially in PD-MCI. The cortical thickness of NBM/ Ch4-innervated regions in PD-MCI patients, including the left posterior cingulate gyrus, insula lobe, parietal lobe, and frontal lobe, and the right posterior cingulate gyrus, parietal lobe, and frontal lobe, were positively correlated with NBM/Ch4 volume loss. The right medial orbitofrontal lobe, anterior cingulate gyri, posterior cingulate gyri, and precuneus lobe were susceptible to cholinergic deficits in PD-MCI patients, while only the right medial orbitofrontal and anterior cingulate were susceptible in PD-NC patients. Our findings contribute to a better understanding of the association between the cortex and NBM/Ch4 in the early stage of PD, especially in different cognitive states. In the future, structural imaging of the NBM/Ch4 could be a new method to predict cognitive impairment in PD.

\section{Acknowledgments}

The authors thank all the subjects who kindly agreed to participate in this study and Kai Liu for the suggestion of the study design.

Funding: This work was supported by the following grants: National Natural Science Foundation of China (no. 82071419); Guangzhou Municipal People's Livelihood Science and Technology Project (no. 201803010085); Highlevel Hospital Construction Project (No. DFJH201907); Supporting Research Funds for Outstanding Young Medical Talents in Guangdong Province (no. KJ012019442); Medical Research Fund of Guangdong Province, China (no. A2017317); National Natural Science Foundation of China (no. 81501112); and the National Key R\&D Program of China (no. 2017YFC1310200). 


\section{Footnote}

Conflicts of Interest: All authors have completed the ICMJE uniform disclosure form (available at http://dx.doi. org/10.21037/qims-20-444). The authors have no conflicts of interest to declare.

Ethical Statement: All subjects provided written informed consent to the research protocol, which was approved by the Medical Ethics Committee of Guangdong Provincial People's Hospital [no. GDREC2018338H(R1)].

Open Access Statement: This is an Open Access article distributed in accordance with the Creative Commons Attribution-NonCommercial-NoDerivs 4.0 International License (CC BY-NC-ND 4.0), which permits the noncommercial replication and distribution of the article with the strict proviso that no changes or edits are made and the original work is properly cited (including links to both the formal publication through the relevant DOI and the license). See: https://creativecommons.org/licenses/by-nc-nd/4.0/.

\section{References}

1. Postuma RB, Berg D, Stern M, Poewe W, Olanow CW, Oertel W, Obeso J, Marek K, Litvan I, Lang AE, Halliday G, Goetz CG, Gasser T, Dubois B, Chan P, Bloem BR, Adler CH, Deuschl G. MDS clinical diagnostic criteria for Parkinson's disease. Mov Disord 2015;30:1591-601.

2. Aarsland D, Andersen K, Larsen JP, Lolk A, KraghSorensen P. Prevalence and characteristics of dementia in Parkinson disease: an 8-year prospective study. Arch Neurol 2003;60:387-92.

3. Lawson RA, Yarnall AJ, Duncan GW, Breen DP, Khoo TK, Williams-Gray CH, Barker RA, Collerton D, Taylor JP, Burn DJ; ICICLE-PD study group. Cognitive decline and quality of life in incident Parkinson's disease: The role of attention. Parkinsonism Relat Disord 2016;27:47-53.

4. Halliday GM, Leverenz JB, Schneider JS, Adler CH. The neurobiological basis of cognitive impairment in Parkinson's disease. Mov Disord 2014;29:634-50.

5. Aarsland D, Creese B, Politis M, Chaudhuri KR, Ffytche DH, Weintraub D, Ballard C. Cognitive decline in Parkinson disease. Nat Rev Neurol 2017;13:217-31.

6. Kehagia AA, Barker RA, Robbins TW. Cognitive impairment in Parkinson's disease: the dual syndrome hypothesis. Neurodegener Dis 2013;11:79-92.

7. Müller ML, Bohnen NI. Cholinergic dysfunction in Parkinson's disease. Curr Neurol Neurosci Rep 2013;13:377.

8. Mesulam MM. Cholinergic circuitry of the human nucleus basalis and its fate in Alzheimer's disease. J Comp Neurol 2013;521:4124-44.

9. Xu R, Hu X, Jiang X, Zhang Y, Wang J, Zeng X. Longitudinal volume changes of hippocampal subfields and cognitive decline in Parkinson's disease. Quant Imaging Med Surg 2020;10:220-32.

10. Apostolova LG, Beyer M, Green AE, Hwang KS, Morra JH, Chou YY, Avedissian C, Aarsland D, Janvin CC, Larsen JP, Cummings JL, Thompson PM. Hippocampal, caudate, and ventricular changes in Parkinson's disease with and without dementia. Mov Disord 2010;25:687-95.

11. Beyer MK, Larsen JP, Aarsland D. Gray matter atrophy in Parkinson disease with dementia and dementia with Lewy bodies. Neurology 2007;69:747-54.

12. Burton EJ, McKeith IG, Burn DJ, Williams ED, O'Brien JT. Cerebral atrophy in Parkinson's disease with and without dementia: a comparison with Alzheimer's disease, dementia with Lewy bodies and controls. Brain 2004;127:791-800.

13. Hall H, Reyes S, Landeck N, Bye C, Leanza G, Double K, Thompson L, Halliday G, Kirik D. Hippocampal Lewy pathology and cholinergic dysfunction are associated with dementia in Parkinson's disease. Brain 2014;137:2493-508.

14. Braak H, Del Tredici K, Bratzke H, Hamm-Clement J, Sandmann-Keil D, Rüb U. Staging of the intracerebral inclusion body pathology associated with idiopathic Parkinson's disease (preclinical and clinical stages). J Neurol 2002;249 Suppl 3:III/1-5.

15. Mesulam MM, Mufson EJ, Levey AI, Wainer BH. Cholinergic innervation of cortex by the basal forebrain: cytochemistry and cortical connections of the septal area, diagonal band nuclei, nucleus basalis (substantia innominata), and hypothalamus in the rhesus monkey. J Comp Neurol 1983;214:170-97.

16. Teipel SJ, Meindl T, Grinberg L, Grothe M, Cantero JL, Reiser MF, Moller HJ, Heinsen H, Hampel H. The cholinergic system in mild cognitive impairment and Alzheimer's disease: an in vivo MRI and DTI study. Hum Brain Mapp 2011;32:1349-62.

17. Kilimann I, Grothe M, Heinsen H, Alho EJ, Grinberg L, Amaro E Jr, Dos Santos GA, da Silva RE, Mitchell AJ, Frisoni GB, Bokde AL, Fellgiebel A, Filippi M, Hampel H, Kloppel S, Teipel SJ. Subregional basal forebrain atrophy in Alzheimer's disease: a multicenter study. J Alzheimers Dis 2014;40:687-700. 
18. Lee JE, Cho KH, Song SK, Kim HJ, Lee HS, Sohn YH, Lee PH. Exploratory analysis of neuropsychological and neuroanatomical correlates of progressive mild cognitive impairment in Parkinson's disease. J Neurol Neurosurg Psychiatry 2014;85:7-16.

19. Choi SH, Jung TM, Lee JE, Lee SK, Sohn YH, Lee $\mathrm{PH}$. Volumetric analysis of the substantia innominata in patients with Parkinson's disease according to cognitive status. Neurobiol Aging 2012;33:1265-72.

20. Ray NJ, Bradburn S, Murgatroyd C, Toseeb U, Mir P, Kountouriotis GK, Teipel SJ, Grothe MJ. In vivo cholinergic basal forebrain atrophy predicts cognitive decline in de novo Parkinson's disease. Brain 2018;141:165-76.

21. Schulz J, Pagano G, Fernandez Bonfante JA, Wilson H, Politis M. Nucleus basalis of Meynert degeneration precedes and predicts cognitive impairment in Parkinson's disease. Brain 2018;141:1501-16.

22. Barrett MJ, Sperling SA, Blair JC, Freeman CS, Flanigan JL, Smolkin ME, Manning CA, Druzgal TJ. Lower volume, more impairment: reduced cholinergic basal forebrain grey matter density is associated with impaired cognition in Parkinson disease. J Neurol Neurosurg Psychiatry 2019;90:1251-6.

23. Gargouri F, Gallea C, Mongin M, Pyatigorskaya N, Valabregue R, Ewenczyk C, Sarazin M, Yahia-Cherif L, Vidailhet M, Lehericy S. Multimodal magnetic resonance imaging investigation of basal forebrain damage and cognitive deficits in Parkinson's disease. Mov Disord 2019;34:516-25.

24. Wei H, Kong M, Zhang C, Guan L, Ba M; for Alzheimer's Disease Neuroimaging I. The structural MRI markers and cognitive decline in prodromal Alzheimer's disease: a 2-year longitudinal study. Quant Imaging Med Surg 2018;8:1004-19.

25. Hanganu A, Bedetti C, Degroot C, Mejia-Constain B, Lafontaine AL, Soland V, Chouinard S, Bruneau MA, Mellah S, Belleville S, Monchi O. Mild cognitive impairment is linked with faster rate of cortical thinning in patients with Parkinson's disease longitudinally. Brain 2014;137:1120-9.

26. Pereira JB, Svenningsson P, Weintraub D, Bronnick K, Lebedev A, Westman E, Aarsland D. Initial cognitive decline is associated with cortical thinning in early Parkinson disease. Neurology 2014;82:2017-25.

27. Segura B, Baggio HC, Marti MJ, Valldeoriola F, Compta Y, Garcia-Diaz AI, Vendrell P, Bargallo N, Tolosa E, Junque C. Cortical thinning associated with mild cognitive impairment in Parkinson's disease. Mov Disord 2014;29:1495-503.

28. Hwang KS, Beyer MK, Green AE, Chung C, Thompson PM, Janvin C, Larsen JP, Aarsland D, Apostolova LG. Mapping cortical atrophy in Parkinson's disease patients with dementia. J Parkinsons Dis 2013;3:69-76.

29. Zarei M, Ibarretxe-Bilbao N, Compta Y, Hough M, Junque C, Bargallo N, Tolosa E, Marti MJ. Cortical thinning is associated with disease stages and dementia in Parkinson's disease. J Neurol Neurosurg Psychiatry 2013;84:875-81.

30. Pagonabarraga J, Corcuera-Solano I, Vives-Gilabert Y, Llebaria G, García-Sánchez C, Pascual-Sedano B, Delfino M, Kulisevsky J, Gómez-Ansón B. Pattern of regional cortical thinning associated with cognitive deterioration in Parkinson's disease. PLoS One 2013;8:e54980.

31. Bohnen NI, Albin RL, Muller ML, Petrou M, Kotagal V, Koeppe RA, Scott PJ, Frey KA. Frequency of cholinergic and caudate nucleus dopaminergic deficits across the predemented cognitive spectrum of Parkinson disease and evidence of interaction effects. JAMA Neurol 2015;72:194-200.

32. Klein JC, Eggers C, Kalbe E, Weisenbach S, Hohmann C, Vollmar S, Baudrexel S, Diederich NJ, Heiss WD, Hilker R. Neurotransmitter changes in dementia with Lewy bodies and Parkinson disease dementia in vivo. Neurology 2010;74:885-92.

33. Lanskey JH, McColgan P, Schrag AE, Acosta-Cabronero J, Rees G, Morris HR, Weil RS. Can neuroimaging predict dementia in Parkinson's disease? Brain 2018;141:2545-60.

34. Cantero JL, Zaborszky L, Atienza M. Volume Loss of the Nucleus Basalis of Meynert is Associated with Atrophy of Innervated Regions in Mild Cognitive Impairment. Cereb Cortex 2017;27:3881-9.

35. Kilimann I, Hausner L, Fellgiebel A, Filippi M, Wurdemann TJ, Heinsen H, Teipel SJ. Parallel Atrophy of Cortex and Basal Forebrain Cholinergic System in Mild Cognitive Impairment. Cereb Cortex 2017;27:1841-8.

36. Emre M, Aarsland D, Brown R, Burn DJ, Duyckaerts C, Mizuno Y, Broe GA, Cummings J, Dickson DW, Gauthier S, Goldman J, Goetz C, Korczyn A, Lees A, Levy R, Litvan I, McKeith I, Olanow W, Poewe W, Quinn N, Sampaio C, Tolosa E, Dubois B. Clinical diagnostic criteria for dementia associated with Parkinson's disease. Mov Disord 2007;22:1689-707; quiz 837.

37. Chen KL, Xu Y, Chu AQ, Ding D, Liang XN, Nasreddine ZS, Dong Q, Hong Z, Zhao QH, Guo QH. Validation of the Chinese Version of Montreal Cognitive Assessment 
Basic for Screening Mild Cognitive Impairment. J Am Geriatr Soc 2016;64:e285-90.

38. Goetz CG, Stebbins GT, Tilley BC. Calibration of unified Parkinson's disease rating scale scores to Movement Disorder Society-unified Parkinson's disease rating scale scores. Mov Disord 2012;27:1239-42.

39. Litvan I, Goldman JG, Troster AI, Schmand BA, Weintraub D, Petersen RC, Mollenhauer B, Adler CH, Marder K, Williams-Gray CH, Aarsland D, Kulisevsky J, Rodriguez-Oroz MC, Burn DJ, Barker RA, Emre M. Diagnostic criteria for mild cognitive impairment in Parkinson's disease: Movement Disorder Society Task Force guidelines. Mov Disord 2012;27:349-56.

40. Gong YX. Revision of Wechsler's Adult Intelligence Scale in China. Acta Psychologica Sinica. 1983. Available online: https://psycnet.apa.org/record/1984-22187-001

41. Gong Y, Wang D, Deng J. Handbook of Wechsler Memory Scale-Revised (WMS-RC), Chinese Version. Changsha: Bulletin of Hunan Medical College, 1989.

42. Ashburner J. A fast diffeomorphic image registration algorithm. Neuroimage 2007;38:95-113.

43. Eickhoff SB, Stephan KE, Mohlberg H, Grefkes C, Fink GR, Amunts K, Zilles K. A new SPM toolbox for combining probabilistic cytoarchitectonic maps and functional imaging data. Neuroimage 2005;25:1325-35.

44. Zaborszky L, Hoemke L, Mohlberg H, Schleicher A, Amunts K, Zilles K. Stereotaxic probabilistic maps of the magnocellular cell groups in human basal forebrain. Neuroimage 2008;42:1127-41.

45. Ashburner J. Computational anatomy with the SPM software. Magn Reson Imaging 2009;27:1163-74.

46. Mufson EJ, Presley LN, Kordower JH. Nerve growth factor receptor immunoreactivity within the nucleus basalis (Ch4) in Parkinson's disease: reduced cell numbers and co-localization with cholinergic neurons. Brain Res 1991;539:19-30.

47. Shimada H, Hirano S, Shinotoh H, Aotsuka A, Sato K, Tanaka N, Ota T, Asahina M, Fukushi K, Kuwabara S, Hattori T, Suhara T, Irie T. Mapping of brain acetylcholinesterase alterations in Lewy body disease by PET. Neurology 2009;73:273-8.

48. Whitehouse PJ, Hedreen JC, White CL 3rd, Price DL. Basal forebrain neurons in the dementia of Parkinson disease. Ann Neurol 1983;13:243-8.

49. Hong JH, Jang SH. Neural pathway from nucleus basalis of Meynert passing through the cingulum in the human brain. Brain Res 2010;1346:190-4.

50. Uribe C, Segura B, Baggio HC, Abos A, Marti MJ,
Valldeoriola F, Compta Y, Bargallo N, Junque C. Patterns of cortical thinning in nondemented Parkinson's disease patients. Mov Disord 2016;31:699-708.

51. Uribe C, Segura B, Baggio HC, Abos A, Garcia-Diaz AI, Campabadal A, Marti MJ, Valldeoriola F, Compta Y, Tolosa E, Junque C. Cortical atrophy patterns in early Parkinson's disease patients using hierarchical cluster analysis. Parkinsonism Relat Disord 2018;50:3-9.

52. Williams-Gray CH, Evans JR, Goris A, Foltynie T, Ban M, Robbins TW, Brayne C, Kolachana BS, Weinberger DR, Sawcer SJ, Barker RA. The distinct cognitive syndromes of Parkinson's disease: 5 year follow-up of the CamPaIGN cohort. Brain 2009;132:2958-69.

53. Bohnen NI, Koeppe RA, Minoshima S, Giordani B, Albin RL, Frey KA, Kuhl DE. Cerebral glucose metabolic features of Parkinson disease and incident dementia: longitudinal study. J Nucl Med 2011;52:848-55.

54. Nie K, Zhang Y, Huang B, Wang L, Zhao J, Huang Z, Gan R, Wang L. Marked N-acetylaspartate and choline metabolite changes in Parkinson's disease patients with mild cognitive impairment. Parkinsonism Relat Disord 2013;19:329-34.

55. González-Redondo R, Garcia-Garcia D, Clavero P, GascaSalas C, Garcia-Eulate R, Zubieta JL, Arbizu J, Obeso JA, Rodriguez-Oroz MC. Grey matter hypometabolism and atrophy in Parkinson's disease with cognitive impairment: a two-step process. Brain 2014;137:2356-67.

56. Alexander GE, DeLong MR, Strick PL. Parallel organization of functionally segregated circuits linking basal ganglia and cortex. Annu Rev Neurosci 1986;9:357-81.

57. Ko JH, Antonelli F, Monchi O, Ray N, Rusjan P, Houle S, Lang AE, Christopher L, Strafella AP. Prefrontal dopaminergic receptor abnormalities and executive functions in Parkinson's disease. Hum Brain Mapp 2013;34:1591-604.

58. Chung SJ, Yoo HS, Lee YH, Lee HS, Ye BS, Sohn $\mathrm{YH}, \mathrm{Kwon} \mathrm{H}$, Lee PH. Frontal atrophy as a marker for dementia conversion in Parkinson's disease with mild cognitive impairment. Hum Brain Mapp 2019;40:3784-94.

59. Baldermann JC, Hardenacke K, Hu X, Koster P, Horn A, Freund HJ, Zilles K, Sturm V, Visser-Vandewalle V, Jessen F, Maintz D, Kuhn J. Neuroanatomical Characteristics Associated With Response to Deep Brain Stimulation of the Nucleus Basalis of Meynert for Alzheimer's Disease. Neuromodulation 2018;21:184-90.

60. Koulousakis P, Andrade P, Visser-Vandewalle V, Sesia T. The Nucleus Basalis of Meynert and Its Role in Deep 
Brain Stimulation for Cognitive Disorders: A Historical Perspective. J Alzheimers Dis 2019;69:905-19.

61. Mak E, Su L, Williams GB, Firbank MJ, Lawson RA, Yarnall AJ, Duncan GW, Owen AM, Khoo TK, Brooks DJ, Rowe JB, Barker RA, Burn DJ, O'Brien JT. Baseline and longitudinal grey matter changes in newly diagnosed Parkinson's disease: ICICLE-PD study. Brain 2015;138:2974-86.

62. Schneider CB, Donix M, Linse K, Werner A, Fauser M, Klingelhoefer L, Lohle M, von Kummer R, Reichmann H, consortium L, Storch A. Accelerated Age-Dependent Hippocampal Volume Loss in Parkinson Disease With

Cite this article as: Rong $\mathrm{S}, \mathrm{Li} \mathrm{Y,} \mathrm{Li} \mathrm{B,} \mathrm{Nie} \mathrm{K,} \mathrm{Zhang} \mathrm{P,}$ Cai T, Mei M, Wang L, Zhang Y. Meynert nucleus-related cortical thinning in Parkinson's disease with mild cognitive impairment. Quant Imaging Med Surg 2021;11(4):1554-1566. doi: 10.21037/qims-20-444
Mild Cognitive Impairment. Am J Alzheimers Dis Other Demen 2017;32:313-9.

63. Uchida Y, Kan H, Sakurai K, Arai N, Kato D, Kawashima S, Ueki Y, Matsukawa N. Voxel-based quantitative susceptibility mapping in Parkinson's disease with mild cognitive impairment. Mov Disord 2019;34:1164-73.

64. Gao Y, Nie K, Mei M, Guo M, Huang Z, Wang L, Zhao J, Huang B, Zhang Y, Wang L. Changes in Cortical Thickness in Patients With Early Parkinson's Disease at Different Hoehn and Yahr Stages. Front Hum Neurosci 2018;12:469. 


\section{Supplementary}

Table S1 Neuropsychological battery

\begin{tabular}{lccc}
\hline Neuropsychological battery & PD-NC & PD-MCl & P value \\
\hline Animal fluency test & $15.12(3.86)$ & $13.80(4.18)$ & 0.225 \\
Digit span & $11.13(2.39)$ & $11.06(2.31)$ & 0.911 \\
Digit symbol & $11.65(2.41)$ & $10.17(2.62)$ & $0.003^{*}$ \\
Logical memory & $5.38(2.64)$ & $3.57(1.34)$ & $0.003^{*}$ \\
Immediate memory & $7.71(2.85)$ & $3.40(3.56)$ & $<0.001^{*}$ \\
Vocabulary & $10.48(2.38)$ & $9.94(2.92)$ & 0.16 \\
Similarities & $11.10(2.52)$ & $10.0(2.76)$ & 0.062 \\
Block design & $9.90(2.21)$ & $9.2(2.84)$ & 0.11 \\
Object assembly & $8.71(1.94)$ & $6.51(1.96)$ & $<0.001^{*}$ \\
\hline
\end{tabular}

Data are mean (standard deviation). Mann-Whitney $U$ test was used to determine significant difference.

Table S2 Region with correlation between the volume of NBM/Ch4 and cortical thickness in PD-MCI

\begin{tabular}{|c|c|c|c|c|c|c|c|}
\hline Cluster no. & p-Max $(-\log 10)$ & Size $\left(\mathrm{mm}^{2}\right)$ & \multicolumn{3}{|c|}{ MNI coordinates } & NVtxs & Annotation of peak region \\
\hline 1 & 3.624 & $1,391.97$ & -4 & -16 & 39 & 3,784 & Left posterior cingulate, \\
\hline 2 & 3.097 & $1,406.28$ & -33 & 17 & 13 & 3,880 & Left pars opercularis \\
\hline 3 & 2.908 & $1,682.5$ & -18 & -74 & 30 & 3,023 & Left precuneus \\
\hline 5 & 4.256 & $3,151.13$ & 20 & -77 & 35 & 7,203 & Right superior parietal \\
\hline 6 & 2.908 & $1,561.18$ & 15 & 35 & 18 & 2,956 & Right superior frontal \\
\hline
\end{tabular}

p-Max, max indicates the maximum -log10 (P value) in the cluster; NVtxs, number of vertices in cluster.

Table S3 Region with significant difference of correlation coefficients between the volume of NBM/Ch4 and cortical thickness in comparison of $\mathrm{PD}-\mathrm{MCI}$ versus $\mathrm{HC}$ and $\mathrm{PD}-\mathrm{NC}$ versus $\mathrm{HC}$

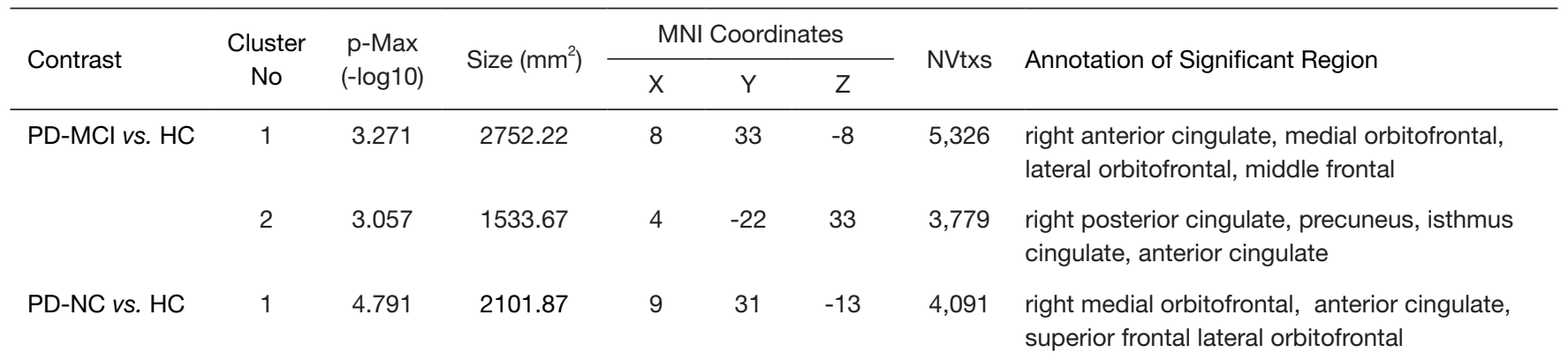

p-Max, max indicates the maximum -log10 (p-value) in the cluster; NVtxs, number of vertices in cluster. 